\title{
LEITES FERMENTADOS COMERCIAIS: ESTUDO COM CONSUMIDORES
}

\section{Commercial fermented milk: study with consumers}

\author{
Michelle Antunes de Alvarenga e Silva ${ }^{l}$, Keila de Matos Silva ${ }^{l}$, Marlon Dias de Castro ${ }^{l}$, \\ Maria Clara Saraiva Souza ${ }^{\prime}$, Paloma Caroline de Lima Campos ${ }^{I}$, \\ Larissa de Oliveira Ferreira Rocha ${ }^{I^{*}}$
}

\begin{abstract}
RESUMO
Entender como os consumidores percebem os produtos alimentícios é fundamental. Além de saber o quanto os consumidores gostam de um produto, as empresas de alimentos precisam de informações sobre quais características sensoriais os consumidores esperam encontrar no produto. Como os consumidores nem sempre conseguem dizer por que gostam de um produto, o teste Check All That Apply (CATA) pode ser uma alternativa simples para obter uma visão da percepção do consumidor frente a um produto. O objetivo do presente trabalho foi aplicar o teste CATA simultaneamente com um teste de aceitação, com a finalidade de entender quais características são importantes para o produto em estudo. Cinco marcas comerciais de leite fermentado foram avaliadas por um painel de 60 consumidores. Os consumidores responderam ao teste CATA contendo 15 atributos que descreviam as características sensoriais do leite fermentado e ao teste de aceitação através de uma ficha de escala hedônica de nove pontos. Os dados do CATA foram avaliados estatisticamente pelo teste Q de Cochran e pela Análise de Correspondência. Houve diferença significativa $(\mathrm{p}<0,05)$ entre as amostras para todos os atributos analisados. A amostra 657 foi a mais aceita pelos consumidores. As características apresentadas por esta amostra foram gosto doce, sabor característico de leite fermentado e cor esbranquiçada. Os atributos que não tiveram impacto na aceitação foram: sabor e aroma de baunilha, coloração caramelo, aspecto coagulado, gosto amargo e ácido. Os resultados apresentados podem ser
\end{abstract}

1 Universidade Federal dos Vales do Jequitinhonha e Mucuri, Instituto de Ciência e Tecnologia, Campus JK, Rodovia MGT 367, km 583, 5.000, Alto da Jacuba,39100-000, Diamantina, MG, Brasil. E-mail: larissa.rocha@ict.ufvjm.edu.br

* Autor para correspondência

Recebido / Received: 15/07/2019

Aprovado / Approved: 30/10/2019 
aplicados pelas indústrias de laticínios para o desenvolvimento de formulações visando atender às expectativas dos consumidores.

Palavras-chave: análise sensorial; Check All That Apply; produto lácteo.

\begin{abstract}
Understanding how consumers perceive food products is crucial. Besides knowing how much they like a product, food companies need information about the sensorial characteristics that people expect to find in a product. As consumers are not always able to explain the reason they like a product, the Check All That Apply (CATA) test can be a simple alternative to obtain information about their perception of a product. This work had the purpose of applying the CATA and the Acceptance test, simultaneously, intending to understand the characteristics of a given product that are more important for consumers. A panel of 60 consumers evaluated five brands of fermented milk. The participants answered the CATA test that presented 15 attributes describing the sensorial characteristics of the fermented milk. For the acceptance test, the hedonic scale of nine points was used. The CATA data were statistically analyzed by the Cochran's Q test and the Correspondence Analysis. There was a significant difference $(\mathrm{p}<0,05)$ between the samples for all the attributes analyzed. The sample 657 was the most accepted by consumers. This sample presented a sweet taste, peculiar flavor of fermented milk and whitish color. The attributes that did not present any impact on the acceptance were flavor and vanilla aroma, caramel coloring, coagulated appearance and bitter and acid taste. The results of this work can be applied in dairy industries to develop new formulations to meet the consumers' expectations.
\end{abstract}

Keywords: sensory analysis; Check All That Apply; dairy product.

\section{INTRODUÇÃO}

Segundo a Instrução Normativa $n^{\circ} 46$, de 23 de outubro de 2007 do Ministério da Agricultura, Pecuária e o Abastecimento (MAPA), o leite fermentado é o produto obtido a partir da fermentação do leite por culturas de bactérias ácido-láticas específicas, cuja fermentação se realiza com um ou vários dos seguintes cultivos: Lactobacillus acidophilus, Lactobacillus casei, Bifidobacterium sp., Streptococus salivarius subsp. thermophilus e/ou outras. Essas devem ser viáveis, ativas e abundantes no produto final e durante toda a vida útil do produto (BRASIL, 2007).

Para leites fermentados a cultura mais utilizada é a de Lactobacillus casei, que compreende bactérias láticas fenotipicamente e geneticamente heterogêneas, aptas a colonizar vários ambientes naturais e criados pelo homem. As bactérias deste grupo têm sido amplamente estudadas com relação a suas propriedades promotoras à saúde. Várias funções benéficas ao organismo humano têm sido atribuídas ao consumo regular de alimentos contendo essas bactérias (BURITI; SAAD, 2007).

O desenvolvimento de novos produtos lácteos fermentados é desafiador devido às altas expectativas e exigência dos consumidores quanto à palatabilidade dos produtos (AKIN; OZCAN, 2017). A escolha do leite fermentado 
pelo consumidor, na maioria das vezes, é influenciada por informações e atributos decisivos, tais como: experiências anteriores, características sensoriais, propriedades nutricionais, cultura de bactérias ácidoláticas, além da marca e gostos pessoais (ARAÚJO et al., 2003; DRANSFIELD et al., 1998).

Portanto, durante o desenvolvimento e a melhoria dos produtos alimentícios, as empresas devem se esforçar para entender os desejos e necessidades dos consumidores, bem como a percepção de seus produtos alimentícios, a fim de garantir o sucesso dos produtos (VARELA et al., 2010). Em estudos sensoriais tradicionais, os testes do consumidor focam na medição hedônica, enquanto a determinação quantitativa das propriedades sensoriais são normalmente realizadas com um grupo de provadores treinados, por análise descritiva quantitativa (ADQ), uma metodologia sensorial descritiva bem estabelecida em vários produtos alimentícios, incluindo laticínios (ALLGEYER et al., 2010; CADENA et al., 2012; GONZALEZ et al., 2011).

No entanto, as técnicas descritivas existentes necessitam de avaliadores treinados, que por consequência aumentam o custo das análises e as tornam longas, devido à necessidade de realizar intensas sessões de treinamento. Como forma de amenizar esses problemas, novas metodologias vêm sendo desenvolvidas, nas quais permitem reunir informações sensoriais dos produtos em tempos mais curtos, visto que essas metodologias podem ser realizadas sem treinamento prévio. Desta forma, essas metodologias se tornam alternativas simples e flexíveis para a avaliação sensorial (CADENA et al., 2014).

A metodologia Check All That Apply (CATA) é descrita como eficiente para descrever e discriminar os produtos, sendo suas principais vantagens a simplicidade e a rapidez com que as análises são efetuadas. Estudos que comparam sua eficiência em relação ao uso de avaliadores treinados relatam altas correlações detectadas entre as avaliações, evidenciando que os consumidores são capazes de avaliar os atributos sensoriais de uma forma semelhante (ARES et al., 2010; BRUZZONE et al., 2012).

O procedimento CATA consiste na elaboração de uma lista de atributos (palavras ou frases) que a equipe de julgadores considera apropriada para descrever os produtos em avaliação. Os produtos são apresentados monadicamente aos julgadores, os quais são solicitados a marcar os atributos que eles consideram presentes naquela amostra específica. O número de atributos selecionados é definido pelo julgador, por seu próprio critério (VALENTIM et al., 2012). A utilização desta técnica na caracterização descritiva de alimentos ainda é recente. Encontra-se na literatura estudos descritivos por meio do CATA para caracterizar sorvete de baunilha (DOOLEY et al., 2010), iogurte probiótico (CRUZ et al., 2013), queijo Minas Frescal (OLIVEIRA et al., 2017), requeijão cremoso (TORRES et al., 2017).

O objetivo do presente trabalho foi aplicar o teste CATA simultaneamente com um teste de aceitação, com a finalidade de entender quais características são importantes em um leite fermentado.

\section{MATERIAL E MÉTODOS}

\section{Leites fermentados}

Cinco marcas comerciais de leite fermentado $(817,526,938,749,657)$, com a especificação "leite fermentado desnatado adoçado", foram adquiridas para o teste sensorial. Os ingredientes, bem como as culturas adicionadas, estão apresentadas na Tabela 1. 


\section{Avaliação sensorial}

A análise sensorial foi realizada com 60 provadores não treinados, de idade variando de 18 a 61 anos, sendo $70 \%$ do sexo feminino, consumidores regulares de leite fermentado. A cada provador foi apresentado o Termo de Consentimento Livre e Esclarecido autorizando a sua participação voluntária na pesquisa. A pesquisa foi aprovada pelo Comitê de Ética, sob número CAAE: 73962117.8.0000.5108.

As análises foram realizadas no Laboratório de Bioquímica de Alimentos da Universidade Federal dos Vales do Jequitinhonha e Mucuri, em cabines individuais sob luz branca. Os provadores receberam $10 \mathrm{~mL}$ dos leites fermentados, na temperatura de refrigeração $\left( \pm 10^{\circ} \mathrm{C}\right)$, em copos descartáveis codificados com números aleatórios de três dígitos acompanhados de um copo com água para limpeza do palato. A apresentação das amostras foi realizada de forma monádica e em blocos completos balanceados (WAKELING; MACFIE, 1995).

\section{Teste "Check-All-That-Apply"}

Antes da realização do teste CATA, foram definidos os termos descritivos em uma sessão com 16 consumidores, onde foram apresentadas duas amostras de leite

Tabela 1 - Ingredientes utilizados no processo de produção dos leites fermentados e cultura utilizada

$\begin{array}{lll}\text { Produto Ingredientes } & \text { Cultura }\end{array}$

Leite desnatado e/ou leite em pó desnatado reconstituído, açúcar, dextrose, preparado laranja cítrica (água, amido 817 modificado, suco de laranja, aromatizante, conservador Não especificada sorbato de potássio e acidulante ácido cítrico), aromatizante, vitaminas $\mathrm{A}$ e D e fermentos lácteos.

Leite desnatado e/ou leite desnatado reconstituído,

526 açúcar, dextrose, fermento lácteo, estabilizante pectina, Lactobacillus casei aromatizante, acidulante ácido cítrico.

Leite desnatado e/ou leite em pó desnatado reconstituído,

938 açúcar, dextrose, aroma idêntico ao natural de baunilha, Não especificada fermento lácteo liofilizado, espessante, pectina.

Leite desnatado e/ou leite desnatado reconstituído, açúcar, Lactobacillus casei glicose, fermento lácteo, aroma.

Shirota

Leite reconstituído desnatado e/ou leite desnatado 657 pasteurizado, xarope de açúcar, açúcar invertido, fermento lácteo, zinco (sulfato de zinco), estabilizante pectina, Lactobacillus aromatizante e edulcorante sucralose. 
fermentado, solicitando que o provador anotasse as similaridades e as diferenças entre ambas em relação aos atributos aparência, aroma, sabor e textura. Os termos descritivos foram gerados através da aplicação de uma ficha de Método Rede (MOSKOWITZ, 1983). Os termos foram selecionados avaliando-se a frequência com que apareciam e, foram eliminados os termos sinônimos ou pouco citados de forma consensual, totalizando 15 termos (gosto ácido, gosto doce, gosto amargo, sabor baunilha, sabor característico de leite fermentado, líquido, leitoso, coagulado, cor caramelo, cor esbranquiçada, brilho, aroma ácido, aroma característico de leite fermentado, aroma adocicado e aroma de baunilha).

Após a definição dos termos descritivos, a ficha do teste CATA foi confeccionada utilizando os 15 termos selecionados de forma balanceada de modo a evitar tendência nos julgamentos. Os provadores foram orientados a marcar na ficha os termos descritivos que descreviam as amostras, de acordo com metodologia descrita por Ares et al. (2011).

\section{Teste de aceitação}

Além do teste CATA, foi realizado simultaneamente um estudo de aceitação das amostras. Cada provador recebeu uma ficha e foi orientado a marcar o grau de gostar ou desgostar do produto em relação à impressão global. Para isso, foram utilizadas fichas com escala hedônica estruturada de nove pontos, cujos extremos correspondem a desgostei extremamente (1) e gostei extremamente (9), conforme Minim (2006).

\section{Análise estatística}

Os resultados do teste de aceitação foram submetidos à Análise de Variância (ANOVA) seguido de teste Scott-Knott ao nível de significância de 5\% através do software Sisvar.
Para o estudo CATA, as frequências de menção de cada palavra foram determinadas pela contagem do número de consumidores que usaram essa palavra para descrever cada leite fermentado. O teste Q de Cochran foi utilizado para cada um dos termos para identificar se houve diferença significativa entre as amostras ao nível de significância de $5 \%$. Os termos significativos foram tratados pela Análise de Correspondência (AC). A AC foi realizada a fim de obter uma representação bidimensional das amostras e a relação entre as amostras e termos. Esta análise foi realizada sobre a tabela de frequência (Tabela 2). As análises foram realizadas pelo software XLSTAT (2018).

\section{RESULTADOS E DISCUSSÃO}

A Tabela 2 demonstra o resultado do teste $\mathrm{Q}$ de Cochran, o qual indicou que houve diferença significativa entre as amostras para todos os atributos avaliados.

Para o questionário CATA aplicado, a primeira e a segunda dimensão da Análise de Correspondência foram responsáveis por $80,70 \%$ da variância dos dados experimentais, sendo $51,57 \%$ e $29,13 \%$, respectivamente (Figura 1).

O gráfico obtido através da Análise de Correspondência mostra a diferenciação entre as amostras. Os consumidores descreveram a amostra 938 como o leite fermentado que apresentou sabor e aroma de baunilha, o que corrobora com os dados apresentados na Tabela 1, que confirma a adição de aroma de baunilha no leite fermentado 938, demonstrando que os consumidores conseguiram identificar o aroma adicionado. A amostra 749 foi caracterizada por apresentar gosto ácido, gosto amargo e cor caramelo. A amostra 817 , conforme os consumidores, apresentou aspecto coagulado e aroma ácido. A amostra 526 caracterizou-se pelo aroma adocicado, aspecto leitoso, líquido e 
brilho, sendo uma amostra intermediária em relação às outras amostras por se aproximar do centro do gráfico. Portanto, estes atributos não foram importantes na diferenciação das amostras.

A amostra 657 apresentou um perfil de cor esbranquiçada, sabor característico de leite fermentado e gosto doce. A amostra 657 foi a única que apresenta sucralose em sua composição (Tabela 1). A sucralose constitui um edulcorante obtido pela cloração da molécula de sacarose nas posições 1', 4' e 6'. A cloração seletiva da sacarose provoca mudanças no seu poder edulcorante, resultando em uma substância 600 vezes mais doce, com perfil sensorial muito semelhante ao da sacarose, sendo possível a detecção de diferença quando a sucralose está em alta concentração (GOLDSMITH; MERKEL, 2001). A presença da sucralose pode ter contribuído para um pronunciamento do gosto doce, sendo este detectado pelos consumidores, conforme apresentado na análise de correspondência (Figura 1).

Tabela 2 - Frequência de escolha de cada atributo por amostra e resultado do teste Q de Cochran para o questionário CATA

\begin{tabular}{|c|c|c|c|c|c|c|}
\hline \multirow{2}{*}{ Atributo } & \multicolumn{5}{|c|}{ Amostras } & \multirow{2}{*}{ p-valor } \\
\hline & 749 & 526 & 817 & 938 & 657 & \\
\hline $\begin{array}{l}\text { Aroma característico } \\
\text { de leite fermentado }\end{array}$ & 26 & 24 & 21 & 20 & 18 & $<0,0004$ \\
\hline $\begin{array}{l}\text { Sabor característico de } \\
\text { leite fermentado }\end{array}$ & 27 & 19 & 21 & 13 & 38 & $<0,0001$ \\
\hline Aroma ácido & 14 & 8 & 6 & 4 & 9 & $<0,0001$ \\
\hline Aroma adocicado & 18 & 26 & 22 & 20 & 18 & $<0,0001$ \\
\hline Aroma de baunilha & 5 & 8 & 19 & 11 & 9 & $<0,0001$ \\
\hline Brilho & 11 & 24 & 16 & 15 & 20 & $<0,0001$ \\
\hline Coagulado & 7 & 4 & 3 & 3 & 3 & $<0,0010$ \\
\hline Cor caramelo & 27 & 21 & 6 & 38 & 22 & $<0,0001$ \\
\hline Cor esbranquiçada & 11 & 23 & 15 & 6 & 29 & $<0,0001$ \\
\hline Gosto ácido & 18 & 13 & 12 & 26 & 14 & $<0,0001$ \\
\hline Gosto amargo & 9 & 8 & 5 & 12 & 5 & $<0,0007$ \\
\hline Sabor baunilha & 9 & 16 & 30 & 12 & 13 & $<00001$ \\
\hline Gosto doce & 17 & 24 & 29 & 18 & 32 & $<0,0001$ \\
\hline Leitoso & 16 & 20 & 15 & 15 & 16 & $<0,0035$ \\
\hline Líquido & 33 & 37 & 34 & 32 & 32 & $<0,0063$ \\
\hline
\end{tabular}

* p-valor maior que o nível de significância alfa $=0,05$ indica que não há diferença significativa. 
As médias de aceitação encontram-se na Tabela 3. A análise de variância demonstrou diferença significativa $(\mathrm{p}<0,05)$ na aceitação das amostras. A amostra 657 apresentouse estatisticamente diferente das demais, obteve a maior média, e, portanto, foi o leite fermentado de maior aceitação sensorial. A média obtida para a amostra 657 situou-se na categoria "gostei moderadamente". As demais amostras situaram-se entre os escores 5 - "indiferente" e 6 - "gostei ligeiramente".

Pode-se verificar que as características apresentadas pela amostra 657 (gosto doce, sabor característico de leite fermentado e cor esbranquiçada) possivelmente foram os atributos responsáveis pela maior aceitação. Observa-se que os provadores tiveram uma menor aceitação pelos leites fermentados que apresentaram sabor e aroma de baunilha, coloração caramelo, aspecto coagulado, gosto amargo e gosto ácido.

Resultados semelhantes foram publicados por Azevedo et al. (2011), os quais avaliaram os atributos sensoriais mais relevantes na aceitação de três marcas comerciais de leite fermentado desnatado adoçado. Segundos os autores, a maior parcela dos consumidores preferiu leite fermentado com baixa acidez e mais doce.

Medeiros et al. (2011) avaliaram a aceitação de três marcas comerciais de leite fermentado, sendo Produto A (Lactobacillus casei defensis), B (Lactobacillus paracasei) e C (Lactobacillus casei e Lactobacillus acidophilus). Os resultados sugeriram uma variabilidade no sabor, correlacionando

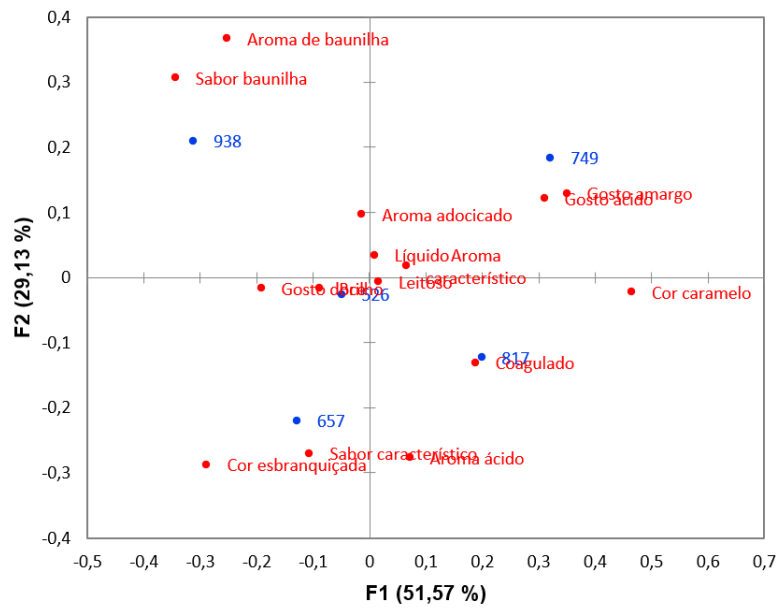

Figura 1 - Representação das amostras e dos termos na primeira e segunda dimensão da Análise de Correspondência da tabela de frequência do questionário CATA

Tabela 3 - Média das notas* obtidas no teste de aceitação

\begin{tabular}{ccccccc}
\hline Amostras & $\mathbf{7 4 9}$ & $\mathbf{5 2 6}$ & $\mathbf{8 1 7}$ & $\mathbf{9 3 8}$ & $\mathbf{6 5 7}$ & $\mathbf{C V}(\mathbf{\%})$ \\
\hline Médias & $5,43^{\mathrm{b}}$ & $5,51^{\mathrm{b}}$ & $5,73^{\mathrm{b}}$ & $5,78^{\mathrm{b}}$ & $6,53^{\mathrm{a}}$ & 35,37 \\
\hline
\end{tabular}

* 1-desgostei extremamente a 9-gostei extremamente.

Médias nas linhas seguidas por letras iguais não diferem entre si, a 5\% de significância, pelo teste de Skott-

Knott. CV\% = coeficiente de variação. 
com o maior teor de acidez e menor $\mathrm{pH}$, e a maior intenção de compra foi para o leite fermentado com Lactobacillus paracasei, o que demonstra que a cultura lática utilizada na produção do leite fermentado também é um fator de escolha do produto, pois influencia no $\mathrm{pH}$ e acidez.

Zacarchenco e Massaguer-Roig (2004) também relataram a influência de diferentes culturas láticas (Streptococcus thermophilus, Bifidobacterium longum e Lactobacillus acidophilus) no $\mathrm{pH}$ e acidez final de leites fermentados. O leite fermentado por Str. thermophilus apresentou a maior acidez e menor $\mathrm{pH}$, enquanto o leite fermentado por Bif. longum apresentou a menor acidez e maior $\mathrm{pH}$.

Os resultados obtidos a partir do teste CATA são interessantes para indústrias de pequeno e médio porte que não possuem infraestrutura financeira para treinar um painel de provadores, podendo auxiliar no desenvolvimento de formulações que se adequem ao perfil dos seus potenciais consumidores.

\section{CONCLUSÃO}

Houve diferença significativa entre as amostras para todos os atributos analisados. A amostra 657 foi a mais aceita pelos consumidores. As características apresentadas por esta amostra foram gosto doce, sabor característico de leite fermentado e cor esbranquiçada. Os atributos que não tiveram impacto na aceitação foram: sabor e aroma de baunilha, coloração caramelo, aspecto coagulado, gosto amargo e ácido. Diante dos resultados obtidos, pode-se verificar quais são os atributos esperados pelos consumidores em um leite fermentado e pela determinação destas características este estudo pode auxiliar no desenvolvimento e/ou melhorias em formulações de leites fermentados.

\section{REFERÊNCIAS}

AKIN, Z.; OZCAN, T. Functional properties of fermented milk produced with plant proteins. Food Science and Technology, v. 86, p. 25-30, 2017.

ALLGEYER, L. C.; MILLER, M. J.; LEE, S. Y. Sensory and microbiological quality of yogurt drinks with prebiotics and probiotics. Journal of Dairy Science, v. 93, p. 44714479, 2010.

ARAÚJO, F. B.; SILVA, P. H. A.; MINIM, V. P. R. Perfil sensorial e composição físico-química de cervejas provenientes de dois segmentos do mercado brasileiro. Ciência e Tecnologia de Alimentos, v. 23, n. 2, p. 121-128, 2003.

ARES, G. et al. Application of a Check-AllThat-Apply question to the development of chocolate milk desserts. Journal of Sensory Studies, v. 25, n. 1, p. 67-86, 2010.

ARES, G., BRUZZONE, F., GIMÉNEZ, A. Is a consumer panel able to reliably evaluate the texture of dairy desserts using unstructured intensity scales? Evaluation of global and individual performance. Journal of Sensory Studies, v. 26, n. 5, p. 363-370, 2011.

AZEVEDO, V. M. et al. Informação nutricional e influência de atributos sensoriais na aceitação de leites fermentados comerciais. Revista do Instituto de Laticínios Cândido Tostes, v. 66, n. 383, p. 49-56, 2011.

BRASIL. Ministério da Agricultura, Pecuária e Abastecimento. Instrução Normativa $n^{\circ}$ 46 , de 23 de outubro de 2007. Regulamento técnico de produção, identidade e qualidade de leites fermentados. Diário Oficial da União: seção 1, Brasília, DF, n. 205, p. 4, 24 out. 2007. 
BRUZZONE, F.; ARES, G.; GIMÉNEZ, A. Consumers' texture perception of milk desserts. II - Comparison with trained assessors' data. Journal of Texture Studies, v. 43, n. 3, p. 214-226, 2012.

BURITI, F. C. A.; SAAD, S. M. I. Bactérias do grupo Lactobacillus casei: caracterização, viabilidade como probióticos em alimentos e sua importância para a saúde humana. Archivos Latino Americanos de Nutricion, v. 57, n. 4, p. 373-380, 2007.

CADENA, R. S. et al. Comparison of rapid sensory characterization methodologies for the development of functional yogurts. Food Research International, v. 64, p. 446-455, 2014.

CADENA, R. S. et al. Reduced fat and sugar vanilla ice creams: Sensory profiling and external preference mapping. Journal of Dairy Science, v. 95, p. 4842-4850, 2012.

CRUZ, A. G. et al. Consumer perception of probiotic yogurt: Performance of check all that apply (CATA), projective mapping, sorting and intensity scale. Food Research International, v. 54, p. 601-610, 2013.

DOOLEY, L. et al. The application of CheckAll-That-Apply (CATA) consumer profiling to preference mapping of vanilla ice cream and its comparison to classical external preference mapping. Food Quality and Preference, v. 21, p. 394-401, 2010.

DRANSFIELD, E.; ZAMORA, F.; BAYLE, M. C. Consumer selection of steaks as influenced by information and price index. Food Quality and Preference, v. 9, n. 5, p. 321-326, 1998.

GOLDSMITH, L. A.; MERKEL, C. M. Sucralose. In: NABORS, L. B. (ed.).
Alternative Sweeteners. 3 ed. New York: Marcel Dekker, 2001. p. 185-208.

GONZALEZ, N. J.; ADHIKARI, K.; SANCHO-MADRIZ，M.F. Sensory characteristics of peach-flavored yogurt drinks containing prebiotics and symbiotics. Food Science and Technology, v. 44, n. 1, p. 158-163, 2011

MEDEIROS, E. J. L. et al. Leite fermentado de marcas comerciais: Estudo da aceitação e correlação com $\mathrm{pH}$ e acidez. Revista do Instituto de Laticínios Cândido Tostes, v. 66, n. 381, p. 46-51, 2011.

MINIM, V. P. R. Análise Sensorial: Estudos com consumidores. Viçosa: Editora UFV, 2006. 225p.

MOSKOWITZ. H. R. Product Testing and Sensory Evaluation of Foods. Westport: Food \& Nutrition Press, 1983. 605p.

OLIVEIRA, E. W. et al. Reformulating Minas Frescal cheese using consumers' perceptions: Insights from intensity scales and Check-AllThat-Apply questionnaires. Journal of Dairy Science, v. 100, n. 8, p. 6111-6124, 2017.

TORRES, F. R. et al. Rapid consumerbased sensory characterization of requeijão cremoso, a spreadable processed cheese: Performance of new statistical approaches to evaluate Check-All-That-Apply data. Journal of Dairy Science, v. 100, n. 8, p. 6100-6110, 2017.

VALENTIN, D. et al. Quick and dirty but still pretty good: A review of new descriptive methods in food science. International Journal of Food Science and Technology, v. 47, n. 8, p. 1563-1578, 2012.

VARELA, P. et al. Influence of brand 
information on consumers' expectations and liking of powdered drinks in central location tests. Food Quality and Preference, v. 21, n. 7, p. 873-880, 2010.

WAKELING, I. N.; MACFIE, H. J. H. Designing consumer trials balanced for first and higher orders of carry-over effect when only a subset of $k$ samples from $t$ may be tested. Food Quality and Preference, v.6, n. 4, p.299-308, 1995.
XLSTAT $^{\circledR}$ Statistical Software for Microsoft Excel $^{\circledR}$. Paris: Addinsoft, 2018. Disponível em: https://www.xlstat.com/

ZACARCHENCO, P. B.; MASSAGUERROIG, S. Avaliação sensorial, microbiológica e de pós-acidificação durante a vida-deprateleira de leites fermentados contendo Streptococcus thermophilus, Bifidobacterium longum e Lactobacillus acidophilus. Ciência e Tecnologia de Alimentos, v. 24, n. 4, p. 674-679, 2004. 